\title{
Blocking endothelial protein C receptor (EPCR) accelerates thrombus development in vivo
}

\author{
Miguel N. Centelles; Cristina Puy1; Jacinto Lopez-Sagaseta1; Kenji Fukudome²; Ramón Montes; José Hermida1 \\ ${ }^{1}$ Division of Cardiovascular Sciences, Laboratory of Thrombosis and Haemostasis, Centre for Applied Medical Research, University of Navarra, Pamplona, Spain; ${ }^{2}$ Department \\ of Immunology, Saga Medical School, Saga, Japan
}

\begin{abstract}
Summary
The endothelial protein C receptor (EPCR) plays an anticoagulant role by improving protein $\mathrm{C}$ activation. Although low levels of activated protein C (APC) constitute a thrombosis risk factor, the relationship between modulating EPCR function and thrombosis has not been addressed so far. Monoclonal antibodies ( $m A b$ ) against murine EPCR were raised, and their ability to block protein C/APC binding was tested. The ferric chloride carotid artery injury model in mice was chosen to test the effect of anti-EPCR mAb on thrombus formation. The time to total occlusion of the vessel was analysed in three groups, given an isotype control mAb (IC), a blocking (RCR-16) or a non-blocking (RCR-20) antiEPCR mAb. RCR-16 prevented the interaction between protein CIAPC and EPCR as demonstrated by surface plasmon resonance and flow cytometry, and inhibited the activation of protein C on the endothelium.
\end{abstract}

IC and RCR-20 were unable to induce such effects. In vivo, RCR-16 shortened the time to total vessel occlusion with respect to IC [13.4 \pm 1.0 (mean $\pm S D$ ) and $17.8 \pm 3.2$ minutes, respectively, $p<0.001]$. Occlusive thrombi lasting for more than one hour were observed in all RCR16 -treated animals, but only in $43 \%$ of IC-treated ones. Results with RCR-20 were indistinguishable from those observed with IC. For the first time, a direct relationship between blocking EPCR and thrombosis is demonstrated. Blocking anti-EPCR autoantibodies can predispose to thrombosis episodes and may constitute a new therapeutic target.

\section{Keywords}

Endothelial cell protein $\mathrm{C}$ receptor, protein C, thrombosis, monoclonal antibodies, animal thrombosis models

\section{Correspondence to:}

Dr. José Hermida

Laboratory of Thrombosis and Haemostasis

Division of Cardiovascular Sciences

Centre for Applied Medical Research (CIMA), University of Navarra

Avenida Pío XII 55, 31008 Pamplona, Spain

Tel.: +34 948194700, Fax: +34 948194716

E-mail: jhermida@unav.es

\section{Financial support:}

This work was supported through the Unión Temporal de Empresas project CIMA and by grants from Instituto de Salud Carlos III (PI051178, Red Temática de Investigación RECAVA RD/0014/0008). Jacinto López Sagaseta was supported by a fellowship from the Education Department, Gobierno de Navarra.

Received: November 5, 2009

Accepted after major revision: February 1, 2010

Prepublished online: March 29, 2010

doi:10.1160/TH09-11-0750

Thromb Haemost 2010; 103: 1239-1244

\section{Introduction}

Endothelial protein C/activated protein $\mathrm{C}$ receptor (EPCR) is an endothelial membrane glycoprotein able to bind protein $\mathrm{C}$ and activated protein C (APC) with high affinity (1). Protein C, when bound to EPCR, is activated more efficiently by the thrombinthrombomodulin complex on the endothelial surface (2,3). APC is a major inhibitor of blood coagulation, and low circulating levels have been related to an increased risk of venous thrombosis (4). Strong evidence supports a role for APC in antiinflammatory/antiapoptotic mechanisms as well (5). EPCR also binds to factor VII/ VIIa (6-8) although the functional consequences of this interaction are currently unknown.

Recently, we and others have demonstrated that blocking antiEPCR autoantibodies can be detected in some patients with thrombosis $(9,10)$. However, somewhat surprisingly, transgenic animals expressing low amounts of EPCR did not display a higher thrombogenic phenotype than their wild-type littermates when their susceptibility to carotid artery thrombosis was examined (11). On the other hand, although it has been demonstrated that baboons challenged with Escherichia coli and blocking antibodies against EPCR developed thrombosis in the microvasculature of a number of organs (12), to the best of our knowledge the effect of EPCR in vivo on macrovascular (arterial or venous) thrombosis development has never been clearly demonstrated. The question thus arises: does EPCR play such an important role that altering its function contributes to thrombosis? Indeed, over-expression of EPCR in mice decreases thrombin generation when the animals are challenged with factor Xa and phospholipids (13). In the present work we provide evidence that blocking EPCR in vivo facilitates macrovascular thrombus formation, most probably due to a reduced ability to generate APC. 


\section{Materials and methods}

\section{Preparation of monoclonal antibodies (mAbs) against murine EPCR}

Since our purpose was to test the role of EPCR in a murine model of thrombosis, $\mathrm{mAbs}$ were raised against murine EPCR according to procedures described elsewhere (14). We selected two mAbs, RCR-16 and RCR-20, to perform our in vivo experiments. Both of them were of IgG2a isotype as determined by a typing kit (The Binding Site, Birmingham, UK). All animal manipulations were approved by the Institutional Animal Care and Use Committee (IACUC) at Saga School of Medicine (Japan).

\section{Biomolecular interaction analysis by surface plasmon resonance (SPR)}

The interaction between recombinant soluble murine EPCR (15) and $\mathrm{mAbs}$ RCR-16 and RCR-20 was studied by SPR in a BIAcore $\mathrm{X}$ Biosensor (BIAcore AB, Uppsala, Sweden). Murine EPCR was immobilised on a CM5 chip (BIAcore) as described elsewhere (15) and mAbs RCR-16 or RCR-20 were injected in a HEPES buffer. Kinetic analyses were performed using BIAEVALUATION software 3.2 RC1 (BIAcore). Murine factor VIIa (kindly gifted by Dr. L.C. Petersen, Novo Nordisk, Maalov, Denmark) was also injected in a HEPES buffer supplemented with $3 \mathrm{mM} \mathrm{CaCl}_{2}$ and $0.6 \mathrm{mM}$ $\mathrm{MgCl}_{2}$ (HEP-Ca-Mg) to check its interaction with murine EPCR.

In a separate set of experiments we studied the ability of RCR-16 and RCR-20 to prevent the binding of APC to murine EPCR. Human APC (Eli Lilly, Indianapolis, IN, USA) was inactivated with biotinylated FPR chloromethylketone (PPACK-b) (Calbiochem, La Jolla, CA, USA). A total of 790 resonance units (RU) of APC-PPACK-b were captured on a streptavidin-coated CM5 chip. Murine EPCR $(100 \mathrm{nM})$ was injected in HEP-Ca-Mg, alone or preincubated with RCR-16, RCR-20 or a rat IgG2a irrelevant $\mathrm{mAb}$ (isotype control) (BD Biosciences, San Jose, CA, USA), and the interaction with APC-PPACK-b was monitored. EDTA $(3 \mathrm{mM})$ was injected at the end of each experiment to eliminate the remains of murine EPCR.

\section{Flow cytometric experiments}

The murine endothelium-derived cell line MS-1 [2279, American Type Culture Collection (ATCC), Manassas, VA, USA], which expresses EPCR and thrombomodulin, was used to test the ability of RCR-16 and RCR-20 to bind to EPCR on the endothelial surface. Both $\mathrm{mAbs}$ and the isotype control were incubated with $1 \times 10^{6} / \mathrm{ml}$ cells. After subsequent incubation with a FITC-labeled anti-rat Ig antibody (BD Biosciences) fluorescence was determined on a FACSCalibur (BD Biosciences). The same cells were used to test the ability of the mAbs to block the binding of APC to the endothelial
A
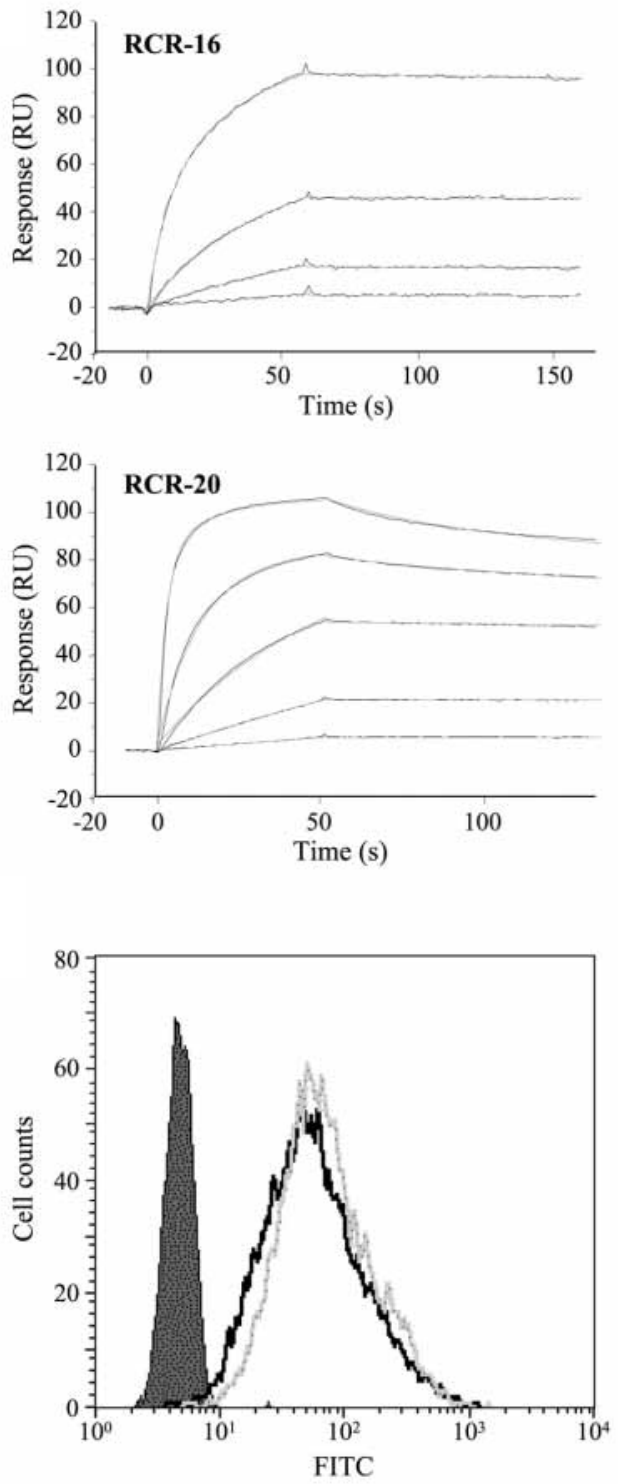

Figure 1: RCR-16 and RCR-20 bind to murine EPCR. A) Murine sEPCR was immobilised on a CM5 chip (BIAcore) and binding of RCR-20 (62.5, 15, $3,0.75,0.18 \mu \mathrm{g} / \mathrm{ml})$ and RCR16 $(62.5,15,3,0.75 \mu \mathrm{g} / \mathrm{ml})$ to murine sEPCR was registered. One representative experiment out of three is shown. $\mathrm{RU}$, resonance units; s, seconds. B) $1 \times 10^{6} / \mathrm{ml}$ MS-1 cells were incubated with 2-20 $\mu \mathrm{g} / \mathrm{ml}$ RCR-16, RCR-20 and isotype control and binding was analysed after subsequent incubation with the appropriate FITC-labelled secondary Ab. Binding was $\mathrm{mAb}$-dose sensitive and did not increase above $10 \mu \mathrm{g} / \mathrm{ml}$. The plot shows the binding of $10 \mu \mathrm{g} / \mathrm{ml}$ RCR-16 (black line), RCR-20 (grey line) and isotype control (grey-filled histogram). One representative experiment out of two is shown.

surface. For this purpose, APC was directly labeled with fluorescein (fl-APC) (2). fl-APC ( $100 \mathrm{nM})$ was incubated with $1 \times 10^{6} / \mathrm{ml} \mathrm{MS-1}$ cells in the presence or absence of $40 \mu \mathrm{g} / \mathrm{ml}$ RCR-16, RCR-20 or isotype control and fluorescence was immediately monitored. 
Non-specific binding of fl-APC to cells was assessed by performing the experiment in the presence of $3 \mathrm{mM}$ EDTA.

\section{APC generation on cultured endothelial cell}

To test the ability of the mAbs to inhibit the APC generation on the endothelial surface, 10,000 MS-1 cells were incubated with 0.02 $\mathrm{U} / \mathrm{ml}$ thrombin (ERL, Swansea, UK) and $50 \mathrm{nM}$ PC (Baxter AG, Vienna, Austria) in the absence or presence of $50 \mu \mathrm{g} / \mathrm{ml} \mathrm{RCR}-16$ or RCR-20. After 30 minutes (min) $0.2 \mu \mathrm{M}$ lepirudin (Schering AG, Berlin, Germany) was added to inhibit thrombin and then the chromogenic substrate S-2366 (Chromogenix, Milan, Italy) was added and its proteolysis by APC kinetically monitored at $405 \mathrm{~nm}$ in a microplate reader (iEMS Reader, Labsystems, Helsinki, Finland).

\section{Murine carotid artery thrombosis model}

Seven- to eight-week-old female C57BL/6 mice were used. A mixture of $100 \mathrm{mg} / \mathrm{kg}$ ketamine and $10 \mathrm{mg} / \mathrm{kg}$ xylazine was applied at the beginning of the experiment and when necessary to keep the animals anaesthetised throughout the experiment. Once the left carotid artery had been carefully exposed, a pulse Doppler flow probe (diameter: $0.5 \mathrm{~mm}$ ) (Transonic, Sidney, Australia) was applied and a baseline carotid artery blood flow measurement was recorded.

We performed an initial dose-finding experiment using a photochemical thrombosis model. In brief, $100 \mathrm{mg} / \mathrm{kg}$ Rose Bengal (Sigma-Aldrich, St Louis, MO, USA) was administered and the common carotid artery illuminated with a $1.5-\mathrm{mW}$ green light $(540 \mathrm{~nm})$ laser (Melles Griot Inc, Carlsbad, CA, USA) until an occlusive thrombus was formed. To test the effect of blocking EPCR on the thrombus development we administered $0,10,20$ or $50 \mu \mathrm{g}$ of RCR-16 1 hour (h) prior to injury. Once a suitable dose was selected, we carefully tested its prothrombotic effect using ferric chloride $\left(\mathrm{FeCl}_{3}\right)$ as a thrombotic stimulus. In brief, a $2 \mathrm{~mm}^{2}$ piece of Whatman paper soaked in a $12 \%$ solution of $\mathrm{FeCl}_{3}$ was applied for 6 min to the adventitial surface of the carotid artery proximal to the probe and blood flow was continuously monitored. Time to carotid artery occlusion was defined as the time from initiation of arterial injury to the onset of total occlusion. Thrombus was considered to be stable if for $1 \mathrm{~h}$ the flow was not recovered above $10 \%$ of that obtained just before applying the stimulus. Mice were i.v. administered $50 \mu \mathrm{g}$ of RCR-16, RCR-20 or isotype control $1 \mathrm{~h}$ prior to injury. The researcher $(\mathrm{MC})$ remained blind regarding the nature of the $\mathrm{mAb}$ injected. All animal manipulations were approved by the IACUC at the Center for Applied Medical Research (Pamplona, Spain).

\section{Statistical analyses}

The differences in times to carotid occlusion between groups were compared using the Kruskal-Wallis followed by the MannWhitney U test.

\section{Results}

\section{mAbs RCR-16 and RCR-20 bind to murine EPCR with high affinity}

A panel of mAbs able to interact with murine EPCR was obtained. Among them, RCR-16 and RCR-20 were selected for the in vivo studies according to their ability or inability to block APC binding (see below).SPR experiments showed a strong interaction between murine EPCR and both RCR-16 and RCR-20 mAbs. Fitting to a bivalent analyte was performed and kinetic constants calculated: $\mathrm{K}_{\mathrm{a}}$ $=5.3 \times 10^{4} \pm 2.0 \times 10^{4}($ mean $\pm \mathrm{SD}, \mathrm{n}=3) \mathrm{M}^{-1} \mathrm{~S}^{-1}, \mathrm{~K}_{\mathrm{d}}=2.1 \times 10^{-4} \pm$ $2.7 \times 10^{-4} \mathrm{~s}^{-1}, \mathrm{~K}_{\mathrm{a} 2}=2,63 \times 10^{-4} \pm 1.8 \times 10^{-4} \mathrm{RU}^{-1} \mathrm{~s}^{-1}, \mathrm{~K}_{\mathrm{d} 2}=4.99 \times 10^{-3} \pm$ $4.97 \times 10^{-3} \mathrm{~s}^{-1}$ for RCR-16 and $\mathrm{K}_{\mathrm{a}}=1.5 \times 10^{5} \pm 0.6 \times 10^{5} \mathrm{M}^{-1} \mathrm{~s}^{-1}, \mathrm{~K}_{\mathrm{d}}=$ $2.2 \times 10^{-2} \pm 2.7 \times 10^{-2} \mathrm{~s}^{-1}, \mathrm{~K}_{\mathrm{a} 2}=4.37 \times 10^{-3} \pm 3.91 \times 10^{-3} \mathrm{RU}^{-1} \mathrm{~s}^{-1}, \mathrm{~K}_{\mathrm{d} 2}=$ $4.35 \times 10^{-3} \pm 4.34 \times 10^{-3} \mathrm{~s}^{-1}$ for RCR-20 (Fig. 1A). Both mAbs were also able to recognise EPCR on the surface of murine endothelial cells as shown by flow cytometry (Fig. 1B).

\section{Murine factor VIla is not a ligand for murine EPCR}

SPR experiments undoubtedly showed that murine factor VIIa was unable to bind to murine EPCR (not shown). Therefore, we did not study the influence of the anti-EPCR mAbs on this potential interaction.

\section{RCR-16 blocks APC binding to murine EPCR}

SPR experiments showed that the binding of murine EPCR to immobilised APC was completely abolished in the presence of RCR-16. However, RCR-20 was not able to block such interaction (Fig. 2A). Accordingly, RCR-16, but not RCR-20, was able to prevent fl-APC binding to murine endothelial cells as shown by flow cytometry (Fig. 2B).

\section{RCR-16, but not RCR-20, impairs protein C activation on the endothelial surface}

Consistently with the previous observations, RCR-16 remarkably reduced down to $49.9 \pm 10.8 \%(n=3)($ mean $\pm S D)$ the thrombininduced APC generation by ms-1 cells. RCR-20 was unable to in- 
A

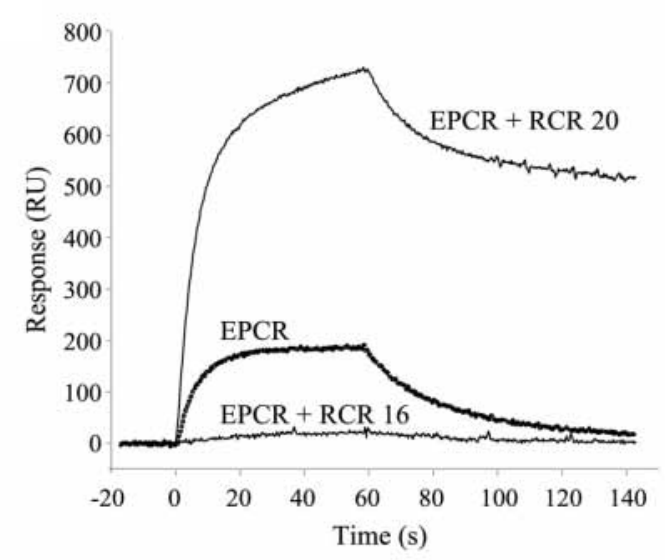

B

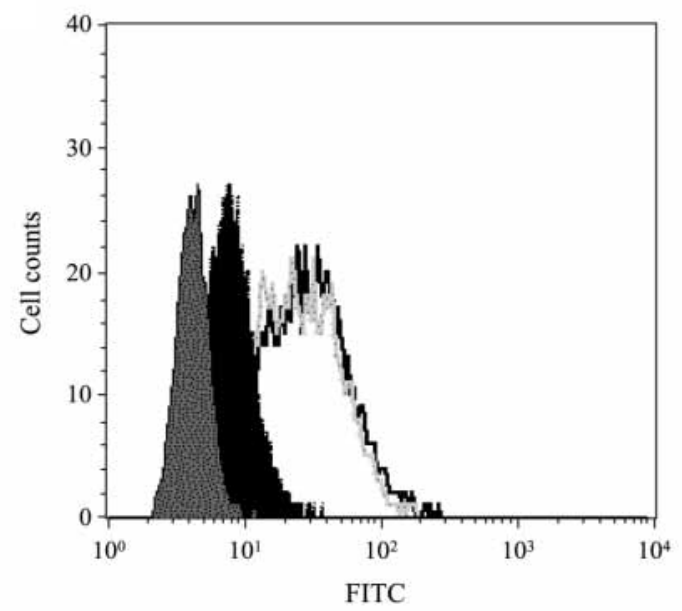

Figure 2: RCR-16, but not RCR-20, prevents the binding of APC to murine EPCR. A) APC-PPACK-b was captured on a streptavidin coated CM5 chip (BIAcore), $100 \mathrm{nM}$ murine EPCR was injected, alone or preincubated with RCR-16 or RCR-20 and the interaction was monitored. RU, resonance units; s, seconds. B) $100 \mathrm{nM}$ fl-APC was incubated with $1 \times 10^{6} / \mathrm{ml} \mathrm{MS-1}$ cells alone (black line) or in the presence of $40 \mu \mathrm{g} / \mathrm{ml} \mathrm{RCR-16}$ (black-filled histogram) or RCR-20 (grey line), and binding was analysed. Binding of fl-APC in the presence of the isotype control was identical to binding of fl-APC alone (not shown). fl-APC was also incubated in the presence of $3 \mathrm{mM}$ EDTA to control for non-specific binding (grey-filled histogram).

fluence protein $\mathrm{C}$ activation. As mentioned above, the affinities of RCR-16 and RCR-20 for murine EPCR are within the same range. Therefore, the different rates of protein $\mathrm{C}$ activation observed with one or another $\mathrm{mAb}$ cannot be explained by differences in their efficiency to displace protein $\mathrm{C}$ from EPCR but rather by the ability of RCR-20 to permit the simultaneous binding, and subsequent activation, of protein $\mathrm{C}$ to EPCR. This property makes RCR-20 a suitable additional control to test the effect of blocking APC generation with RCR-16 on thrombus development in vivo.

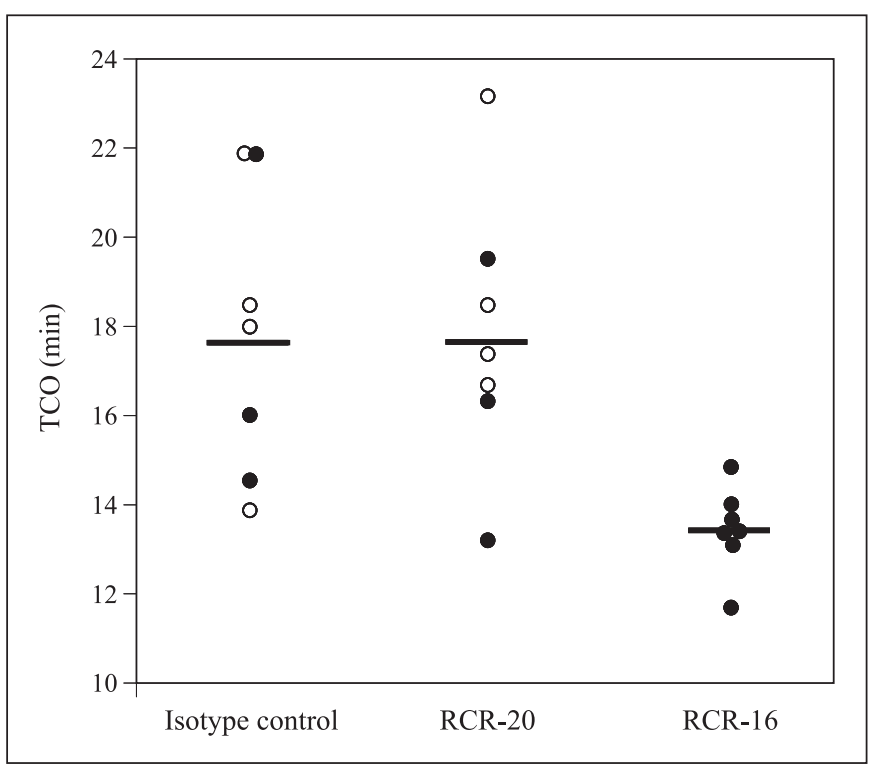

Figure 3: Blocking EPCR accelerates thrombus formation and stabilises the clot upon endothelial insult. Mice were i.v. administered $50 \mu \mathrm{g} /$ ml RCR-16, RCR-20 or isotype control 1 hour prior to $\mathrm{FeCl}_{3}$-induced carotid artery endothelial injury and the time to thrombotic occlusion in the carotid artery downstream of the site of injury was assessed by Doppler flow measurement. Individual times to carotid occlusion are represented by circles. Closed circles represent vessels that were unopened for at least 1 hour after occlusion while open circles represent partially or totally reopened vessels during that time. The mean times to occlusion for each group are represented by horizontal bars. TCO, time to carotid occlusion; min, minutes.

\section{Blocking EPCR does influence the carotid artery occlusion time}

We used the photochemical thrombosis model to perform the RCR-16 mAb dose-finding experiment because it is nicely reproducible and allows to detect small differences better than the $\mathrm{FeCl}_{3}$ model. On the other hand, the last one was used to compare the effect of the blocking and non-blocking anti-EPCR mAbs because this model is particularly useful to monitor the stability of the thrombi. First, we observed a dose-response effect of EPCRblocking RCR-16 mAb in the photochemical thrombosis model. Time to thrombus formation was increasingly shortened as RCR-16 dose increased: $55.83 \pm 15.87$ min (mean \pm SD) $(n=6)$; $43.33 \pm 12.86 \mathrm{~min}($ mean $\pm \mathrm{SD})(\mathrm{n}=6) ; 33.66 \pm 8.83 \mathrm{~min}($ mean \pm $\mathrm{SD})(\mathrm{n}=6) ; 33.56 \pm 5.07$ min $($ mean \pm SD $)(\mathrm{n}=6)$ when administered $0,10,20$ or $50 \mu \mathrm{g}$ of RCR-16, respectively. Thus, doses above $20 \mu$ g guaranteed maximum effect. We decided to administer $50 \mu \mathrm{g}$ of RCR-16 in the $\mathrm{FeCl}_{3}$ thrombosis model. In such case, a significant difference in the time required to reach the onset of total occlusion between mice previously administered RCR-16 and mice administered the isotype control $\mathrm{mAb}$ was observed [13.4 \pm 1.0 $\min ($ mean $\pm S D)(n=7)$ and $17.8 \pm 3.2 \min (n=7)$, respectively, $\mathrm{p}<0.001]$. However, the non-blocking mAb RCR-20 was not able 
to accelerate the thrombus formation when compared with the isotype control treatment $[17.8 \pm 3.1 \mathrm{~min}(\mathrm{n}=7)]$. Additionally, occlusive thrombi lasting for more than $1 \mathrm{~h}$ were observed in all the animals treated with RCR-16, while only three out of seven mice treated with RCR-20 or the isotype control developed stable thrombi $(\mathrm{p}<0.001)$ (Fig. 3). Blocking EPCR must therefore induce an earlier and more stable thrombus after endothelial insult. Importantly, the fact that the non-blocking anti-EPCR mAb-treated animals behaved like the isotype control-treated ones suggests that the prothrombotic effect of RCR-16 relies on its ligand blocking property.

\section{Discussion}

For the first time, direct evidence is provided showing that blocking the ligand binding site of EPCR in vivo accelerates arterial thrombus formation. Furthermore, the thrombi formed under these conditions are more resistant to spontaneous lysis. Among the raised mAbs exhibiting a high affinity for murine EPCR, RCR-16 was chosen to block EPCR function. First, it efficiently abolished the binding of APC to murine EPCR as shown by SPR. Second, as assessed by flow cytometry, blocking was also observed with murine endothelial cells i.e. RCR-16 prevented the binding of APC to cell membrane EPCR. Importantly, RCR-16 reduced the ability of thrombin to activate protein $\mathrm{C}$ on the surface of endothelial cells. These properties make this mAb particularly suitable for our purposes. RCR-16 undoubtedly reduced the time required to total occlusion of the injured vessel, and helped to form a more stable clot as well. In order to rule out the possibility that these effects were induced by triggering the complement system or by endothelial activation, we also studied the times to carotid occlusion in the presence of RCR-20, an anti-EPCR mAb which, although exhibiting a similar affinity for EPCR than RCR-16, does not prevent protein C/APC from binding to the receptor. The fact that the behaviour of the mice given RCR-20 was undistinguishable from that observed in the animals administered the isotype control permits us to conclude that the effect of RCR-16 was due to the specific blocking of the EPCR binding site.

The most likely explanation for the prothrombotic effect of RCR-16 in vivo lies in its ability to prevent protein $\mathrm{C}$ activation. Previous literature supports the view that an important decrease in APC generation takes place in vivo upon EPCR blocking (3). Indeed, the well-known thrombotic tendency observed in individuals with protein $\mathrm{C}$ deficiency and the relationship found between low circulating APC levels and thrombosis risk further substantiate this hypothesis $(4,16,17)$. Recently, we and others discovered that human factor VII/VIIa binds to human EPCR with similar affinity than protein $\mathrm{C}(6-8)$. Although the functional consequences of this interaction in vivo are presently unknown, one could speculate that preventing factor VII from interacting with EPCR could influence thrombus development. However, at least in the present study this is not the case: murine factor VIIa was undoubtedly un-

\section{What is known about this topic?}

- Blocking protein C-EPCR interaction decreases circulating activated protein $C$ levels generated in response to thrombin in baboons.

- Over-expression of EPCR in mice decreases thrombin generation when the animals are challenged with factor $\mathrm{Xa}$ and phospholipids.

- The autoantibodies against EPCR have been associated with thrombosis in humans.

\section{What does this paper add?}

- We generated monoclonal antibodies against murine EPCR.

- This study demonstrates that blocking EPCR in vivo promotes thrombosis development.

able to bind to murine EPCR as checked by SPR. This lack of interaction, surprising at first sight, can be explained by the fact that a residue of the Gla domain which is critical for interaction with EPCR, i.e. Phe at position 4 (18), is substituted in murine factor VII by Leu.

Transgenic animals expressing low amounts of EPCR are not particularly prone to develop thrombosis upon $\mathrm{FeCl}_{3}$-induced endothelial insult (11). This finding would seem to be in contradiction to our results. One explanation could be that those transgenic animals expressed small but sufficient amounts of EPCR to prevent thrombosis. Alternatively, a role played by some compensatory mechanism due to the genetic manipulation in those animals cannot be ruled out. A direct prothrombotic effect of RCR-16 in our models would provide an explanation as well, although it seems unlikely since the non-blocking mAb, RCR-20, did not exert any effect. In any case, our results are consistent with a large number of scientific evidences supporting the hypothesis that EPCR plays a leading role in the protein $C$ anticoagulant system $(12,19)$.

We and others have detected the presence of blocking antiEPCR autoantibodies in patients with thrombosis, which, once purified, were able to reduce the activation of protein $\mathrm{C}$ on endothelial cells in vitro $(9,10)$. We now provide direct evidence that blocking anti-EPCR antibodies exacerbates thrombosis in animal models, thus strongly suggesting that autoantibodies may play an active role in human thrombotic pathology.

In conclusion, for the first time the direct involvement of EPCR in preventing thrombosis in vivo is demonstrated. The occurrence of high titers of blocking anti-EPCR autoantibodies in some patients could explain their clinical episodes of thrombosis. We suggest that strategies aimed to identify and clear these autoantibodies may be therapeutically useful.

\section{Acknowledgements}

We thank Eva Molina and Maider Esparza for their excellent technical assistance. 


\section{References}

1. Fukudome K, Esmon CT. Identification, cloning, and regulation of a novel endothelial cell protein C/activated protein C receptor. J Biol Chem 1994; 269: 26486-26491.

2. Stearns-Kurosawa DJ, Kurosawa S, Mollica JS, et al. The endothelial cell protein C receptor augments protein $\mathrm{C}$ activation by the thrombin-thrombomodulin complex. Proc Natl Acad Sci USA 1996; 93: 10212-10216.

3. Taylor FB Jr, Peer GT, Lockhart MS, et al. Endothelial cell protein C receptor plays an important role in protein C activation in vivo. Blood 2001; 97: 1685-1688.

4. España F, Vayá A, Mira Y, et al. Low level of circulating activated protein C is a risk factor for venous thromboembolism. Thromb Haemost 2001; 86: 1368-1373.

5. Mosnier LO, Zlokovic BV, Griffin JH. The cytoprotective protein C pathway. Blood 2007; 109: 3161-3172.

6. López-Sagaseta J, Montes R, Puy C, et al. Binding of factor VIIa to the endothelial cell protein $\mathrm{C}$ receptor reduces its coagulant activity. J Thromb Haemost 2007; 5: 1817-1824.

7. Ghosh S, Pendurthi UR, Steinoe A, et al. Endothelial cell protein $\mathrm{C}$ receptor acts as a cellular receptor for factor VIIa on endothelium. J Biol Chem 2007; 282: 11849-11857.

8. Preston RJ, Ajzner E, Razzari C, et al. Multifunctional specificity of the protein C/activated protein C Gla domain. J Biol Chem 2006; 281: 28850-28857.

9. Hurtado V, Montes R, Gris JC, et al. Autoantibodies against EPCR are frequently found in antiphospholipid syndrome and are a risk factor for foetal death. Blood 2004; 104: 1369-1374.

10. Lavigne-Lissalde G, Cochery-Nouvellon E, Granier G, et al. Diffuse skin necrosis in a patient with an anti-endothelial cell protein $\mathrm{C}$ receptor autoantibody which blocks protein C activation. J Thromb Haemost 2005; 3: 413-415.
11. Castellino FJ, Liang Z, Volkir SP, et al. Mice with a severe deficiency of the endothelial protein $\mathrm{C}$ receptor gene develop, survive, and reproduce normally, and do not present with enhanced arterial thrombosis after challenge. Thromb Haemost 2002; 88: 462-472.

12. Taylor FB Jr, Stearns-Kurosawa DJ, Kurosawa S, et al. The endothelial cell protein $\mathrm{C}$ receptor aids in host defense against Escherichia coli sepsis. Blood 2000; 95: 1680-1686.

13. Li W, Zheng X, Gu J, et al. Overexpressing endothelial cell protein C receptor alters the hemostatic balance and protects mice from endotoxin. J Thromb Haemost 2005; 3: 1351-1359.

14. Ye X, Fukudome K, Tsuneyoshi N, et al. The endothelial cell protein $\mathrm{C}$ receptor (EPCR) functions as a primary receptor for protein $\mathrm{C}$ activation on endothelial cells in arteries, veins, and capillaries. Biochem Biophys Res Commun 1999; 259 : 671-677.

15. López-Sagaseta J, Montes R, Hermida J. Recombinant expression of biologically active murine soluble EPCR. Protein Expr Purif 2009; 64: 194-197.

16. Dahlbäck B, Villoutreix BO. Regulation of blood coagulation by the protein C anticoagulant pathway: novel insights into structure-function relationships and molecular recognition. Arterioscler Thromb Vasc Biol 2005; 25: 1311-1320.

17. Dahlbäck B. Advances in understanding pathogenic mechanisms of thrombophilic disorders. Blood 2008; 112: 19-27.

18. Oganesyan V, Oganesyan N, Terzyan S, et al. The crystal structure of the endothelial protein C receptor and a bound phospholipid. J Biol Chem 2002; 277: 24851-24854.

19. Van de Wouwer M, Collen D, Conway EM. Thrombomodulin-protein C-EPCR system: integrated to regulate coagulation and inflammation. Arterioscler Thromb Vasc Biol 2004; 24: 1374-1383. 